\title{
Matices Kogui. \\ Representaciones y negociación en la marginalidad
}

\author{
JuAN CARLOS ORRANTIA \\ Estudiante DE DOCTORAdo Yale University \\ juanorrantia@yahoo.com
}

\begin{abstract}
Resumen
- STE ARTí́culo ANALIZA ALGUNOS ASPECTOS DE LA PRODUCCión DE LA IMAGEN DE LA [ cultura kogui como elemento fundamental para la conservación de la Sierra Nevada de Santa Marta, Colombia. Para ello examina autores y textos antropológicos, así como procesos y herramientas tecnocientíficas propias de la estrategia conservacionista de la Sierra Nevada. En este proceso identifica cómo nociones como las de marginalidad de la Sierra Nevada se han reafirmado entre sus habitantes. Sin embargo, se trata de una marginalidad propuesta como medio de clasificación y como forma de producción cultural y política, la cual es negociable desde, con y por medio de las imágenes que, a su vez, la refuerzan.
\end{abstract}

PALABRAS ClAVE: representaciones antropológicas, discurso de la conservación y herramientas tecnocientíficas, negociación de la marginalidad, indígenas kogui.

Abstract

T HERE EXISTS AN IMAGE OF SO-CALLED KOGUI CULTURE THAT CONSIDERS IT AN ESSENTIAL element for the preservation of biodiversity as part of the conservationist schemes in the Sierra Nevada de Santa Marta (Colombia). My purpose here is to engage the history of this image from certain anthropological representations that participated in its definition, as well as tracing the process of articulation of this image with the discourse of conservation. For these matters I consider the work of authors from the Sierra's ethnographic archive as well as processes and tecno-scientific tools used in the conservationist strategy of the Sierra. As a result of this, notions such as marginality have been reinforced on the Sierra Nevada and its inhabitants. Notwithstanding, my approach to marginality considers it not just a form of classification, but also a process of cultural and political production, that is negotiable from, by, and through the images that reinforce it.

KEY wORDS: Anthropological representations, discourse of conservation and tecno-scientific tools, negotiation of marginality, Kogui indigenous people.

$$
\text { Revista Colombiana de Antropología }
$$

Volumen 38, enero-diciembre 2002, pp. 45-75 
La desorientación producida por el riesgo ambiental contemporáneo se debe, en parte, a una disyunción sistemática entre lo que se le dice a la gente acerca del riesgo y a la forma en que las personas deben enfrentar el manejo del riesgo en sus vidas diarias (...) Una metáfora como la del desarrollo sostenible encubre mensajes contradictorios como un argumento unificado (Kim Fortun, 1999).

\section{INTRODUCCIÓN*}

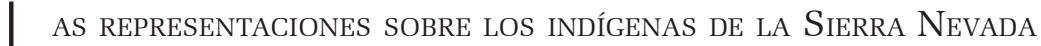
DE Santa Marta (SNSM) no han sido siempre las mismas. De - losrelatos colonialistas de cronistas y viajeros, pasando por el siglo diecinueve cuando resaltaron un Otro incivilizado y al mismo tiempo invisible (Figueroa, I998), los kogui han llegado a etiquetas de café orgánico en mercados internacionales ${ }^{1}$; actualmente, la representación más popularizada se basa en la definición de la llamada cultura kogui como la esencialización de un sistema de pensamiento estrechamente relacionado con la sostenibilidad -global- de la biodiversidad. Esta imagen identifica una asociación entre dos entidades asumidas como homogéneas, la Sierra y la cultura kogui, que se conciben como una unidad mutuamente dependiente y prácticamente simbiótica. En este texto se presenta una lectura crítica de una parte del proceso de la re-producción de esta imagen, a la cual me

* Este artículo está basado en partes de mi tesis de maestría en la Universidad de Georgia y en algunas reflexiones en proceso. Agradezco a Peter Brosius y Monika Therrien, y a los(las) evaluadores de la RCA por sus comentarios a versiones iniciales del texto. Diana Bocarejo y Ángela Rivas se esforzaron especialmente en lograr que la sutileza de sus comentarios no disminuyera la riqueza de sus críticas. Así mismo, agradezco muchísimo a todas las personas con quienes he conversado sobre su paso reciente y no tan reciente por la Sierra; la lista es larga.

I. En Londres puede adquirirse Sanctuary Organic ${ }^{\mathrm{TM}}$. The Legendary Coffee of the Kogi Indians. referiré como (la imagen de) lo kogui, o la representación ideal de los Hermanitos Mayores: una idea occidental sobre gente y lugar.

En 1988 el antropólogo Carlos Uribe llamó la atención sobre esta imagen, exponiendo una idea que muchos admiten pero que ha tendido a difundirse y consolidarse en medio de retóricas ambientalistas: que los habitantes indígenas de la SNSM no son "los escogidos", sino "seres humanos que debieron confrontar y resolver las demandas de su entorno" (Uribe, I988: IO). No obstante, la imagen se mantiene como parte de un proceso complejo de constantes 


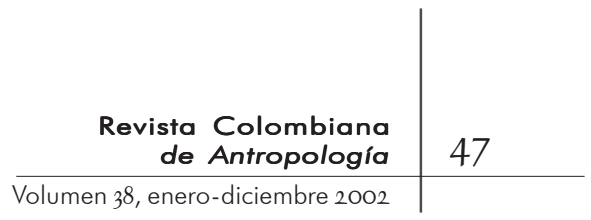

reproducciones $^{2}$. Mi lectura de las mismas se centra en el papel de la antropología en este proceso y en la relación entre las formas de representación antropológica de los indígenas, las nuevas formas biotecnológicas (Escobar, I996, I999; Haraway 1991, 1997) y los procesos burocráticos de la conservación de la biodiversidad.

2. En la actualidad existen varias tendencias que participan en la pro-

Esta lectura me permite una aproxima- ducción de conocimiento e inforción a la construcción de la imagen antro- mación sobre lo kogui, derivadas pológica-ambientalista de lo kogui, por del trabajo de productores culturamedio de sus transformaciones y reacomo- el músico Carlos Vives (véase dos en diferentes contextos. Y digo varios, Orrantia, 2002). La imagen ambienpues mi análisis sobre las representacio- tal de la llamada cultura kogui fornes no está localizado en un solo lugar; se políticos de identidad en las comubasa en recorridos e imaginaciones por tex- nidades indígenas de la Sierra tos antropológicos, entrevistas con antro-

(Ulloa, 200I).

pólogos y antropólogas, conservacionistas, y mis propias observaciones sobre estos diferentes contextos en los que se produce la imagen de lo kogui. De esta manera, mi reflexión crítica se incribe en las corrientes disciplinarias que se desarrollaron en las décadas de 1970 y I980, las cuales han llevado a entender las interpretaciones antropológicas como intervenciones dentro de un canal de representaciones (Marcus y Fischer, I999 [1986]). Por ende, mi trabajo etnográfico pretende ampliar esta identificación de la autoridad del texto antropológico en la producción de dicha imagen, al complementar y complejizar sus articulaciones con otros discursos (Marcus, I999) -como el de la conservación de la biodiversidad-.

Esta aproximación me permite abordar la volatilidad de $1 o$ kogui; algo que aparentemente tomamos y entendemos como una unidad, como un bloque cultural de una forma de pensar y actuar, se transforma en una idea que descomplejiza la producción de conocimiento, así como las formas de interacción entre los discursos y sus interlocutores. Entender lo kogui como algo volátil y flexible, que se desprende de sus connotaciones tradicionales en diferentes momentos, abre nuevos espacios para leer la complejidad cultural que las interacciones, las movilizaciones, la información y las nuevas tecnologías han creado. Por eso utilizo la analogía de los matices, como una gama de variaciones de un color, que cambian, se transforman y adquieren nuevos reflejos. Si bien la imagen parece ser la misma, lo kogui como esa unión entre espacio geográfico y cultura es, al mismo tiempo, 
una cultura antigua, una cultura actual, una técnica de conservación y una yuxtaposición que crea tensión dentro del sistema burocrático de instituciones conservacionistas. En este sentido, lo kogui se manifiesta heterogéneamente y en sus matices podemos leer su volatilidad y sus sutilezas.

\section{UNA NOTA SOBRE MARGINALIDAD}

L PROCESO CONSERVACIONISTA EN LA SiERRA HA SIDO ELABORADO A - partir de la definición de la problemática serrana en términos - que enfatizan sobre la condición de marginalidad de la región y sus pobladores indígenas:

Los diferentes grupos indígenas dominaron el manejo del agua, creando sistemas cuyas características pueden ser admiradas hoy en día en las ruinas de sus ciudades de piedra, caminos y otras construcciones. También desarrollaron un sofisticado sistema social, cultural y espiritual que sobrevive en algunos de sus descendientes (...) En la década de los sesentas el saqueo sistemático de sitios sagrados Tairona también debilitó la tradiciones de los grupos indígenas. En los setentas la introducción de cultivos ilícitos, debido a la demanda internacional, resultó en la pérdida de más de Ioo.000 hectáreas de bosques (...) Este contexto de un conflicto difundido desató un proceso caótico de ocupación de tierras y un uso no sostenible de los recursos naturales, así como la continuada violación a los derechos humanos. A esto se le sumó el desplazamiento de los grupos indígenas hacia las partes altas del macizo. En I99o solo el I8\% de la cobertura de bosques originales permanecía, con los consecuentes y adversos efectos sobre la disponibilidad del agua (Fundación Pro Sierra Nevada de Santa Marta, s. f.).

Este tipo de diagnósticos regularizan a los habitantes indígenas y las ideas que tenemos de ellos y ellas, y de su papel en un proceso de prevención del riesgo y de conservación cultural y biológica de un área ambiental estratégica. Así mismo, implican la condición de marginalidad como parte del proceso de degradación -ambiental y cultural- de la Sierra. Como trasfondo del proceso de deterioro ambiental se asume que las personas que habitan allí han sido abandonadas por el estado y se encuentran alejadas de los centros de poder, lo cual ha constituido un factor importante en la determinación de su condición actual. En 


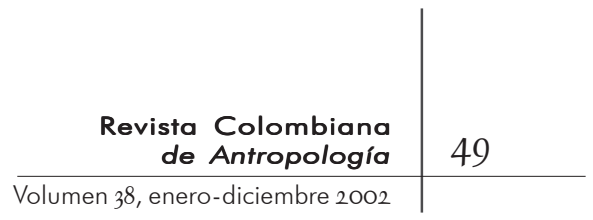

este sentido, la marginalidad se entiende como una forma de abandono que crea condiciones irregulares para atender las necesidades de servicios de sus pobladores en salud, educación, etcétera. Así, la marginalidad se equipara y reduce popularmente al aislamiento, aquí se asume una idea de marginalidad que incluye estas connotaciones tradicionales -también ligadas a procesos de desigualdad-, pero mi intención es descentralizar esa marginalidad situándome como observador desde la marginalidad ${ }^{3}$.

Así, la marginalidad no puede verse simplemente como el resultado de movimientos expansionistas o de divisiones político-económicas sino como una producción cultural y política. De esto se desprende que, más que una característica de algún grupo de personas, forma parte de los procesos de producción cultural de las mismas ${ }^{4}$; se convierte así en un elemento que surge de procesos históricos y políticos, y es negociable-en términos culturales-. Esto permite cuestionar las representaciones, conceptos e imágenes que sustentan definiciones de la problemática ambiental (de la SNSM) como la expuesta más arriba y se entiendan no sólo como realidades evidentes -ipor supuesto que los bosques están desapareciendo!-, sino también como resultados contingentes de procesos históricos y políticos en evolución (Gupta y Ferguson, 1997). Tal es mi propósito en este texto.

\footnotetext{
3. Planteamientos como los de Anna Tsing (I993), que cuestionn nociones tradicionales sobre este concepto llaman la atención sobre su volatilidad y flexibilidad

4. En este sentido, la marginalidad se asemeja al concepto de fronteras, como lugares entendidos tradicionalmente como alejados deprimidos, etcétera, pero que han llegado a ser vistos como lugares activos en los que nociones tradicionales de cultura se cuestionan, negocian y acomodan (véase, por ejemplo, Alvarez, I995).
}

\section{Afirmaciones o/cultas DE LA ANTROPOLOGÍA SERRANA}

$\int \mathrm{I}$ I BIEN LAS INTERPRETACIONES ANTROPOLÓGICAS DE LOS PROCESOS sociales y culturales de la Sierra tienen su propia historia de debates y cuestionamientos académicos, el uso del concepto de cultura en la mayoría de ellas ha sido relativamente uniforme. La denominada cultura kogui se define a partir de una serie de conceptos que enfatizan sobre la simbiosis entre lugar, gente y cultura. La figura ancestral de La Madre y la idea indígena de la Fertilidad están en el centro de las representaciones kogui y en 
las antropológicas sobre el pensamiento y la práctica kogui. A su vez, esta dualidad se sustenta en el principio de la tradición ${ }^{5}$, entendida como un concepto que sugiere una relación con el pasado remoto y el patrimonio arqueológico tangible hoy. Así se entiende la cultura como un ente homogéneo, compartido, definible y, en algunos casos, prácticamente atemporal.

Estas concepciones de la cultura como algo con un orden estructural y profundo y reconstruible en su totalidad, homologan cultura con espacio geográfico: los kogui de la Sierra. Por un lado,

5. Para críticas y revisiones importantes sobre el uso de este concepto en la Sierra, véanse Bocarejo (200I) y Uribe (I990). pacial que localiza individuos/grupos en territorios específicos. Este tipo de reducciones recurre con frecuencia a explicaciones simbólicas. En los textos antropológicos sobre el saber de los kogui, la Madre Universal, considerada también como la montaña en sí, es explicada como la personificación y materialización de los principios y poderes espirituales de la deidad:

(...) la Madre Universal, la misma naturaleza se dirigió al hombre y le indicó cómo debía tratarla (...) de esta manera la Madre hizo la "ley" (Reichel-Dolmatoff, I985, I: 240).

Esta relación se define entonces bajo el concepto de fertilidad, que implica una perpetuación, un renacer constante de espíritu y materia:

La religión Kogui se basa en el culto de la fertilidad. Las expresiones concretas de esta fertilidad son la mujer como madre y tierra de cultivo. Mujer y tierra se vuelven así sinónimos, lo mismo como coito y siembra, esperma y semilla, niño y fruto (Reichel-Dolmatoff, I985, II: 79).

La esencia de lo kogui se ha construido así a partir de la combinación de nociones sobre I) una religiosidad o espiritualidad; 2) que controla el manejo del complejo entorno natural;3) heredada de una antigua civilización -tairona-.

Al localizar territorialmente la cultura, la identidad se reduce a una definición que involucra categorías geográficas, que si bien cumplen papeles importantes en la producción y definición de 


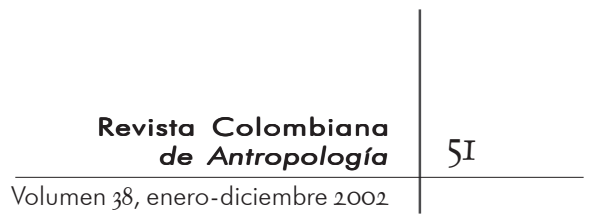

conceptos como territorio, limitan también la movilidad cultural de los individuos localizándolos en(tre) categorías espaciales -como es el caso de la marginalidad-; la cultura queda así contenida en categorías que no permiten ver la flexibilidad histórica de las representaciones y sus articulaciones con otros discursos. Por ende, estas asociaciones de lugar, gente y cultura deben entenderse como creaciones históricas y sociales (Gupta y Ferguson, 1997). Para el caso de la Sierra, esta deconstrucción de la imagen de lo kogui hace visible la subjetividad del proceso de producción de las representaciones y, así mismo, presenta las bases que facilitaron la articulación entre estas y discursos como el de la conservación de la biodiversidad.

\section{Etnografía de la espiritualidad}

F L ÉNFASIS EN LA ESPIRITUALIDAD ESTÁ EN EL CENTRO DE LA OBRA DE

$\sqcup$ Konrad Th. Preuss ${ }^{6}$, y se deriva de su educación en la tradición de la etnología alemana del siglo diecinueve. Su educación con Adolf Bastian hace clara la centralidad del geist "el espíritu, el alma de un pueblo -un espíritu metafísico que se encuentra en el núcleo de una cultura particular-" (Uribe, I993: II). Para poderse acercar al geist de los kogui, Preuss dedicó su permanencia en la Sierra a la recuperación de cantos y mitos para desarrollar una definición de la cultura kogui así:

Un maravilloso mundo de creencias, en el que

6. Si bien resalto la obra de Preuss, debo aclarar que los análisis que giran en torno a la cultura como expresión simbólico-religiosa siguen muy presentes para la Sierra. Sin embargo, considero que trabajos en esta línea como los de Cárdenas (2000) son reinterpretaciones de la imagen central e histórica que extranjeras y frutc irracional hacen soportable y atractiva la cruda realidad de su experiencia, se ha adaptado de analizo. tal manera a la naturaleza de la montaña que parecería que los mitos y cantos sólo se hubieran creado en este suelo (...) Su capacidad de manejar internamente las fuerzas demoníacas de la naturaleza no es lo más notable de su vida espiritual, sino su gran cohesión, cómo todo está fundido unitariamente con su concepción de la Madre Universal y de los primeros sacerdotes humanos, sus antepasados (...) (Preuss, I993: I7-I8).

Bastian había establecido el estudio de la tela del mundo natural y de la mente humana como una entidad unitaria, como una unidad psíquica. Aquel pensador alemán, fundador del museo 
etnológico de Berlín, fusionó ideas de la filosofía alemana del geist con las percepciones positivistas sobre naturaleza y cultura del siglo de las luces bajo la influencia de Von Humboldt (Koepping, 1983). Como tal, la interpretación de Preuss refuerza la idea de un modo de vida que incorpora el medio natural y lo explica en términos religiosos. Así, las expresiones filosóficas alemanas del geist, en versión de Bastian vía Preuss, han contribuido en la interpretación de la imagen de lo kogui como una relación entre religión y medio ambiente, en la que el control de lo material se logra por medio del control de lo metafísico.

\section{Ecología del asombro}

Para mí el evento decisivo fue el encuentro con los Kogi (...) [Aquí] reinaba una actitud intelectual (...) Muchas son las facetas de [su] mundo intelectual, por lo cual para comprender su cosmovisión debemos primero entender sus bases míticas, pues éstas explican la manera como los Indígenas conciben el universo y como se adaptan a éste en términos ecológicos, sociales, filosóficos, y sicológicos (Reichel-Dolmatoff, I99I: 8I-86).

Y así lo hizo el profesor y para su explicación desarrolló un modelo psico-ecológico-cultural que explicaba la cosmovisión kogui. Este encuentro personal y profundamente emotivo con habitantes de la Sierra Nevada fue interpretado por ReichelDolmatoff en términos científicos, en gran parte influenciado por su paso en la etnografía amazónica de las décadas de i970 y I980. En ese periodo se consolidaron en la etnográfica amazónica la historia de un legado francés largo y fuerte con la ecologíacultural estadounidense. La idea central de las interpretaciones surgidas de este contexto era que las adaptaciones humanas al medio ambiente podían entenderse a partir de la identificación de las estructuras culturales-mentales que se expresaban por medio de complejos sistemas de organización social y la actividad ritual (véanse, por ejemplo, Århem, I993; Descola, I996; Jackson, I983; Hugh-Jones [Christine], I979; Hugh-Jones [Stephen], I979; Reichel-Dolmatoff-Dolmatoff, I976a; Viveiros de Castro, 1996). Tras su periodo amazónico, Reichel-Dolmatoff adaptó estas ideas a sus observaciones iniciales entre los kogui; el resultado fue el modelo de la metáfora sexual-alimenticia. 


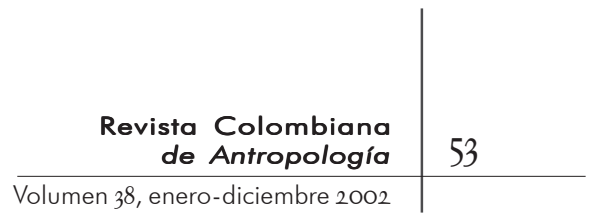

Según Reichel-Dolmatoff, tras la conquista los grupos asentados en las partes bajas de la Sierra se vieron obligados a buscar refugio en las partes altas, lo cual obstaculizó el antiguo sistema de acceso a productos costeros. Esto generó un fuerte cambio en los patrones de subsistencia, transformando un sistema de agricultura de irrigación intensiva en uno basado en productos mixtos -muchos de los cuales eran introducidos y ricos en almidones, es decir, malos nutritivamente-. Los kogui se adaptaron entonces a este desequilibrio agroalimentario por medio de un sistema de pensamiento basado en el conocimiento astronómico de las estaciones, que, junto con un sistema de regulación de la organización social -Tuxes y Dakes- controlaba el acceso de las diferentes familias o linajes a los distintos nichos -ecosistemas- de la Sierra. Para Reichel-Dolmatoff este sistema complejo de adaptación ecológica está descrito en el pensamiento de los mamas, quienes son los encargados de su control y preservación (Reichel-Dolmatoff, 1975, I976a, I976b, I978, I982a, I982b; Uribe, I988).

7. Para reflexiones sobre esta relación entre la antropología serrana y el activismo, véanse, por ejemplo, Bocarejo (200I); Dussán de ReichelDolmatoff (1965); Giraldo (1999); Piles debe entenderse también dentro de los contextos políticos en que se produjeron ${ }^{7}$. Estas teorías y modelos de las ciencias sociales se convertirían así en razonamientos académicos para soportar una lucha desde la antropología -necesaria por demás en su momento- por el reconocimiento y valoración de las comunidades indígenas; pero de la misma manera, al equiparar cultura y medio ambiente la lucha por la defensa del uno llevaba a la lucha por la defensa del otro. No en vano el establecimiento del movimiento conservacionista de la Sierra se apoyó fuerneda (200I); Uribe (I980).
8. Es necesario aclarar que los mo- vimientos activistas de organizacio- nes indígenas que hoy por hoy utilizan explicaciones similares presentan argumentos distintos para sus pro- pias luchas y la defensa por el me- dio ambiente. Estos discursos políticos son textos complejos y si bien a veces se relacionan con re- presentaciones antropológicas no pueden reducirse a estas. Mi punto aquí está en demostrar la relación que existe entre las representaciones antropológicas y el establecimiento del movimiento ambientalista en la Sierra Nevada. temente en este tipo de explicaciones ${ }^{8}$.

\section{Pasado y presente}

En la elevada grandeza de la SNSM viven los sobrevivientes delo que una vez fue una poderosa tribu. Estos indios de la montaña son 
llamados Aurohuacas ${ }^{9}$ y son gente tan particular que difieren completamente de todos los otros que he visto cerca del mar Caribe, y deben ser los remanentes de una gente muy antigua y particular, y mucho más desarrollada, que en algún tiempo remoto fueron obligados a tomar refugio en las partes altas de la montaña debido a las presiones y la violencia de vecinos mucho más salvajes (Nicholas, I9OI: 637).

Uno de los grandes mitos en la antropología de la Sierra identifica a los kogui como los herederos de los habitantes prehispánicos del norte de la Nevada. La construcción de grandes terrazas,

9. Aunque el nombre Arhuaco corresponde a otro grupo étnico actual de la SNSM, en la época en que se escribió este texto este nombre se utilizaba de manera general para todas las poblaciones indígenas en la SNSM. Nicholas describe su significado como compuesto de las palabras Oro y Huaca, lo cual vendría a significar algo así como oro-escondido, imaginario muy recurrente en las zonas de la vertiente norte de la Sierra. caminos y ciudades de piedra le otorgó a aquellos, los tairona, la categoría de civilización. En este esquema de descendencia, la complejidad que caracterizó la civilización de estos últimos se ha transformado: su expresión material imponente dio paso a una más espiritual. Esta última ha llegado a representar una forma de poder siempre y cuando cumpla con el atributo que se lee en la fórmula: entre más tradicional sea el conocimiento, más fiel a su origen, más puro y, por ende, más potente.

Esta idea se ha expresado en diversas formas. Por ejemplo, en la obra de Gregory Mason (I940), un arqueólogo estadounidense quien visitó a los kogui en búsqueda de pruebas que afirmaran la continuidad tairona-kogui, se expresa como una magia antigua y poderosa. Con cierta dosis de sarcasmo, Mason describe relatos de colombianos espantados por una magia kogui, oscura, prístina y misteriosa. Inicialmente lo atribuyó a supersticiones incultas y relacionadas con el gusto de los colombianos por el alcohol. Sin embargo, a medida que fue internándose en el mundo místico y tradicional de los kogui fue creyendo cada vez más en esta magia, al punto de terminar convencido de su existencia. La magia kogui era tan poderosa que lograba convencer al pensamiento occidental (Mason, I940; véase Uribe, I998).

Actualmente, la fuerza de la tradición es entendida en la magia/sabiduría del manejo ecológico kogui de los ecosistemas serranos, idea que emerge en gran parte de los resultados del proyecto arqueológico Ciudad Perdida (véase infra). A partir de estudios sobre la arquitectura, la ingeniería y los ecosistemas de 


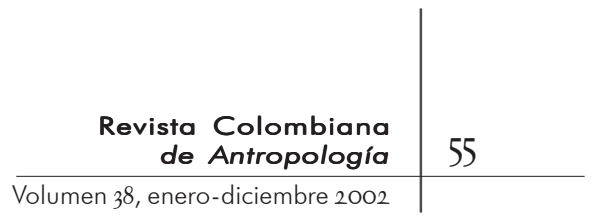

los sitios arqueológicos de la Sierra, apoyados también en interpretaciones etnográficas, muchas de las cuales se basaron en la obra de Reichel-Dolmatoff (véase Legast, 1986), se concluyó que la forma en que los sitios monumentales se relacionaban con el entorno natural era evidencia de una cultura que había logrado un equilibrio y manejo sostenible del medio ambiente (véanse Giraldo, 1999; Herrera de Turbay, 1985; Serje, I986; Soto, I988b). Así, se popularizó el concepto de tradición para ligar históricamente a la actual cultura kogui con la llamada cultura tairona por medio del manejo (sostenible) del medio ambiente.

Recientemente, Diana Bocarejo (200I) ha propuesto que para algunos mamas en la Sierra la tradición es un concepto flexible que se adapta a nuevas situaciones, personas y materiales, en una re-transformación constante. Esta muestra de flexibilidad y la autoría (de sujetos particulares) en el discurso indígena, que a su vez sugiere la subjetividad en la autoría de las interpretaciones clásicas de la antropología serrana y sus relaciones con la idea de tradición y el pasado tairona.

Entonces, la descripción de Nicholas, el relato de Mason y la información que obtuvieron etnógrafos y arqueólogas de sus informantes y de sus lecturas de la obra de Reichel-Dolmatoff para sustentar sus resultados, plantean la permanencia histórica de un diálogo entre los indígenas serranos y la antropología. Espiritualidad, ecología y tradición forman así un complejo de articulaciones, un juego de traducciones y representaciones que se asemeja a la formación de redes de conocimiento en las que las interpretaciones se articulan en una red de representaciones, políticas, y el mundo de los descubrimientos científicos, creando narrativas compuestas (Latour, 1993 $)^{10}$. Por consiguiente, el manejo, la articulación y la traducción de estos tres conceptos, que juntos definen la esencia de lo kogui, sugiere también que la reinterpretación ha sido, y es, múltiple, y forma parte del conocimiento producido y trasmitido (por indígenas y antropólogos). Este es un proceso que se reelabora constantemente, que, como pretendo mostrar en el resto del artículo, se mezcla y confunde en las formas en que la imagen de lo kogui se ha relacionado con el discurso de la conservación de la biodiversidad.

IO. En el contexto de la literatura de viajeros y de textos etnográficos, la situación se asemeja también al concepto de Pratt (I992) de autoetnografía. Sin embargo, una lectura a las formas de expresión y acomodación indígenas en la literatura antropológica en este contexto, si bien está relacionada con esta discusión, merece por sí sola un análisis el cual escapa de los límites de este trabajo. 


\section{LOS BOSQUES BUROCRÁTICOS DE LA CONSERVACIÓN}

OS IDEALES INDIGENISTAS DE LA DEFENSA DE LOS INDIO, SU CULTURA Y su terrtorio son difíciles de lograr. Sin embargo, en el caso de la SNSM estos ideales han logrado consolidarse y convertirse en mecanismos de acción por medio de las organizaciones no gubernamentales (ONG) que mediante representaciones esencialistas y románticas, con profundas raíces en la antropología, se articulan con los aparatos ambientalistas transnacionales (Brosius, I997, 200I).

En su análisis de la historia del indigenismo en Brasil, Alcida Ramos (I998) llamó la atención sobre las complicaciones del proceso de articulación del movimiento conservacionista y el indígena, principalmente por el choque entre ideales y burocracia. Al ir creciendo, las ONG se ven envueltas en procesos de profesionalización y burocratización, que dejan poco espacio para la Otredad -indígena- que se resiste a la domesticación burocrática. Como resultado, los individuos indígenas y los ideales románticos de sus protectores se vuelven incompatibles con la lógica funcional de la burocracia. Entonces, la única forma que tienen de sobrevivir y permanecer es por medio del simulacro ${ }^{11}$;

II. Ramos utiliza para su análisis el concepto de simulacro desarrollado por Baudrillard. indígenas a las formas y necesidades de la organización. Este sujeto es el que Ramos ha llamado el indígena hiperreal: un indio perfecto cuyas virtudes, sufrimientos y estoicismo inagotables le han merecido el derecho a ser defendido por los profesionales de los derechos indígenas (Ramos, 1998: 276).

En la Sierra Nevada de Santa Marta este proceso de construcción de un Otro ecológico homogéneo por parte del movimiento conservacionista fue el resultado del contacto e interrelación entre antropólogos y ambientalistas que se consolidaron durante la década de 1970. Los informes publicados en la época son muy pocos, por lo cual la fuerza que lo kogui tuvo en la producción técnica de los jóvenes antropólogos y activistas de ese periodo no logra el estatus de narrativa científica como 


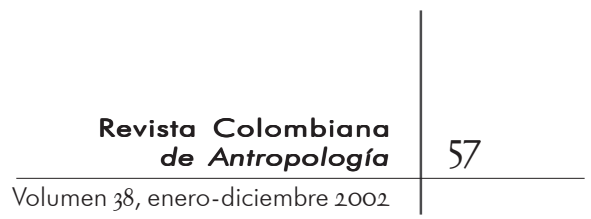

la de épocas anteriores. Por este motivo, para el análisis de este periodo me apoyo en algunas conversaciones con personajes del periodo de activismo al que me he referido, considerados por algunos como románticos e idealistas. Estos jóvenes de los años 1970 fueron los visionarios y gestores de lo que hoy se conoce como el movimiento para la conservación de la Sierra Nevada de Santa Marta.

Su historia tiene como eje central el desarrollo de proyectos de diversa índole en el sitio conocido hoy como Ciudad Perdida. A mediados de la década de 1970, bajo la dirección del antropólogo Álvaro Soto se estableció un gran pro-

yecto arqueológico en la zona del alto I2. Para reflexiones sobre este Buritaca ${ }^{12}$. El mismo formaba parte de la ini- proyecto, véase Giraldo (Ig99). ciativa nacional de las estaciones antropológicas del Instituto Colombiano de Antropología. Su objetivo principal era establecer presencia institucional y científica de los antropólogos y la antropología en áreas apartadas o marginales del país (Soto, I975), con el fin de fomentar y promover un nacionalismo que tuviera en cuenta las regiones, los lugares y las culturas -y sitios monumentales- que se encontraban en áreas marginales (entrevista a Álvaro Soto, junio de 200I).

El proyecto se desarrolló en tres etapas. Durante las dos primeras se hizo la mayor parte del trabajo arqueológico in situ y se reconstruyeron las estructuras líticas principales. Actualmente, es curioso ver las interpretaciones que se han hecho sobre la relación entre esas estructuras y los bosques de su entorno. Las fotos que vi en el apartamento de Soto, en las que las estructuras líticas de Ciudad Perdida están libres de vegetación, se yuxtaponen y articulan con lo que he visto en mis viajes a ese lugar. Pero, en todo caso, "los bosques han estado siempre, ¿o no?" Esto se lo preguntó él mismo para explicarme un momento crucial en el desarrollo del proyecto, la llamada tercera etapa, cuando se incluyó "el componente ecológico" y se decidió expandir el proyecto a otros sitios de las cuencas del Buritaca y Guachaca:

- ¿Cómo entender el bosque sin sus gentes, y cómo entender sus gentes sin entender el bosque?; la cultura como un todo integrado con el ambiente natural. Una visión holística que separa la verdadera antropología de un simple acto de malabarismo.

Según él, y en parte definida por las lecciones del profesor Reichel-Dolmatoff, esta definición determinó su cambio de 
estrategia y la preocupación por los bosques.

El avance de la frontera agrícola y la devastación forestal por parte de los cultivadores de marihuana llevó a Soto y a los miembros de su grupo a buscar mecanismos y emprender acciones de conservación para estos lugares. Bajo esas premisas y circunstancias, los lugares arqueológicos se convirtieron, más que en centros de investigación arqueológica en sí, en lugares y puestos de control para el conocimiento y la protección ambiental (Vollmer, I983). La antropología se combinó con estudios ecológicos y biológicos, y la autoridad se reforzó con la presencia del ejército y la policía. Así, se combinaron la autoridad del conocimiento científico por parte de académicos y la autoridad estatal en estas regiones marginales.

Entendido en el contexto de la época, cuando la antropología colombiana se había fragmentado entre una tendencia activista, políticamente comprometida, y otra de corte científico, positivista (Uribe, 1990), los jóvenes involucrados en este proyecto vieron en él una forma de acceder, por medio de la investigación, a la defensa de la diversidad cultural y ambiental. Esto ha sido claro en conversaciones con personas comprometidas con el proyecto en todas sus fases ${ }^{13}$. Pero de acuerdo con las conversaciones que sostuve con Sylvia Botero, una de las etnógrafas en la tercera etapa, la tensión y la

I3. Antropólogas y antropólogos como Ana María Groot de Mahecha, Luisa Fernanda Herrera de Turbay, José Luis Mahecha, Guillermo Rodríguez, Carlos Uribe, y participantes y trabajadores del proyecto como Manuel Carabalí Amú, Franky Rey y Ricardo Rey entre otros.

llevó a institucionalizarlo: búsqueda de cambios en este proceso fue muy clara. Hablar de la Sierra y de su paso por ella le generan entusiasmo y emoción, recuerdos de asombro y nostalgia. La fuerza de la imagen kogui no sólo les hizo vivir momentos inolvidables sino que les permitió sentir un ideal -por más romántico y momentáneo que fuere-. Y esto los

En ese momento cambiamos toda la estructura de [el proyecto] Ciudad Perdida y toda la mirada; nosotros pensamos que no se podía excavar, y prohibimos las excavaciones. Decidimos que daba lo mismo un arqueólogo que un guaquero. Entonces empezaron a llegar los indígenas y nos dimos cuenta que ellos eran los sabios del lugar, y no el ripio del lugar. Cuando ellos llegaban empezamos a aprender kogui, qué era $\mathrm{CP}$, qué podíamos hacer por CP, cómo podíamos conocer el mundo kogui, y nos empezaron a enseñar, y empezamos a aprender mitos en 


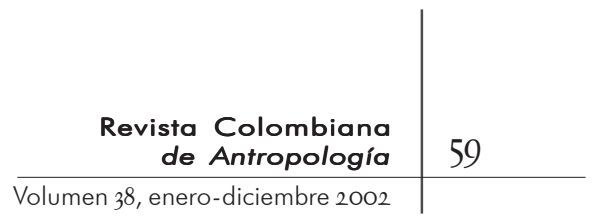

kogui, empezaron a ponernos aseguranza, hicimos un rancho kogui (...) empezamos a formar con los indígenas un frente anti-guaquería. Nosotros, bajo la selva, nos dimos cuenta de que esta es muy frágil y de una [gran] riqueza, una exhuberancia; y nos dimos cuenta que si se movía la selva se movía todo lo sagrado; empezamos a entender que había algo sagrado.

Entonces en Ciudad Perdida nosotros nos inventamos un concepto que se llamaba El parque histórico y la reserva natural del alto río Buritaca.

Representaciones y sentimientos similares lograron ser difundidas por los medios, y así se hicieron públicas y tomaron fuerza las interpretaciones de los kogui como herederos de este complejo sistema de pensamiento. Encontraron eco en ideas indigenistas que se venían gestando, y fueron difundidas por la prensa nacional en artículos con títulos como "Réquiem por la Sierra Nevada", "Indígenas de Sierra Nevada se extinguen”, "Foro indígena para proteger ciudad sagrada” (véanse, El Espectador, 1968, 1982, 1983; El Tiempo, I97I, 1976a, I976b; Hurtado, I984; Soto, 1988a). La idea de que estos ecosistemas y la cultura kogui estaban amenazados se popularizó, y así, se combinaron representaciones antropológicas con ideas de riesgo y amenaza. Como resultado se categorizó al lugar y a sus habitantes como un patrimonio en peligro, que debía protegerse.

Este proceso, en el que se articularon las representaciones antropológicas sobre los kogui y el movimiento ambiental naciente, se reforzó a partir de un proceso regional de articulaciones entre intereses de fuerzas económicas y sociales, y el discurso de la tradición (Coronel, 2000) ${ }^{14}$. Así mismo, permitió la articulación de la Sierra con procesos globales por medio de la introducción del discurso conservacionista. De esta manera, la Sierra y lo kogui se incluyeron como parte de un territorio global en términos ambientales, y se clasificó dentro de la categoría de riesgo transnacional, como riesgo de la pérdida

I4. Desde una economía política crítica, Coronel ha cuestionado la idea de marginalidad como aislamiento regional que se maneja en el discurso conservacionista para la Sierra Nevada. Para ella, lo regional en términos posmodernos no puede establecerse únicamente a partir de la ausencia de capitalización y creación de valores, sino también del hecho de la incapacidad en cuanto a toma de decisiones -en especial sobre seguridad colectiva y la administración de flujos financieros-. En consecuencia, la Sierra ha sido constituida en una localidad de la geopolítica global-por medio de su incorporación al discurso conservacionista y los flujos de capitales transnacionales- en tanto posmodernismo periférico -en cuanto a las contradicciones que surgen de la revaloración posmoderna de nuevos poderes como la identidad (tradicionalismo) y su verdadera representación, la cual, en últimas, es retomada por las ONG- (2000: IO9-IIO). 
de la biodiversidad. Este proceso ha enriquecido las metanarrativas de una sociedad que ya se veía en riesgo, y plantea la necesidad de modelos sofisticados y regímenes regulatorios para prevenir el mismo (Fischer, 1999).

La proclamación de la Sierra Nevada como reserva de la biosfera a partir de su inclusión en el programa hombre y la biosfera de la Unesco en 1978 dio origen a la utilización de estos modelos ${ }^{15}$. La aplicación de esta figura de la conservación internacional fue apoyada desde la antropología por el profesor Reichel-Dolmatoff quien reclamó "la urgente necesidad de establecer a la Sierra Nevada como una reserva de la biosfera (...) donde los kogui serían protegidos contra las influencias destructivas, mientras la investigación a largo plazo podría llevarse a cabo por grupos de especialistas en ecología, geografía, botánica ...” (Reichel-Dolmatoff, I982a: 45). De igual modo, la creación de la reserva de la biosfera dio paso más adelante al establecimiento de la ONG Fundación Pro Sierra Nevada de Santa Marta (FPSNSM).

El proyecto Ciudad Perdida llegó a su fin en I984, tras la disolución de la Fundación Tairona, última entidad a su cargo. Sus miembros más activos quedaron desvinculados temporalmente. Algunos se recluyeron en la Sierra, otros permanecieron en Santa Marta o en Bogotá, pero en todo caso continuaron con su propósito de conocer mejor la Sierra y encontrar soluciones para su conservación.

I5. Esta figura de la conservación internacional está basada en el principio de que "un equilibrio entre metas aparentemente contradictorias, como la conservación de la biodiversidad, el desarrollo económico y social, y la preservación de valores culturales asociados, es posible" (Unesco, 200I).

I6. "El área núcleo debe ser legalmente establecida para garantizar protección a largo plazo sobre el paisaje, los ecosistemas y las especies contenidas en ella (...) Normalmente el área núcleo no debe estar sujeta a actividades humanas, excepto por la investigación, el monitoreo, y en caso dado, a la extracción por medios tradicionales para uso de las comunidades locales" (Unesco, 200I. Énfasis mío).
El gobierno de Belisario Betancur propuso la creación de una ONG para atender los problemas de conservación y manejo de la Sierra. Esta iniciativa fue acogida por el fotógrafo del proyecto, Juan Mayr, junto con otros miembros del proyecto entre los que se encontraban Ricardo Rey, Sylvia Botero y Guillermo Rodríguez. Así nació la Fundación Pro Sierra Nevada de Santa Marta. Con la creación de esta entidad el vínculo entre el discurso conservacionista y la historia de representaciones antropológicas sobre la Sierra Nevada se institucionalizó.

El gobierno nacional le encomendó como primera tarea a la Fundación la im- 


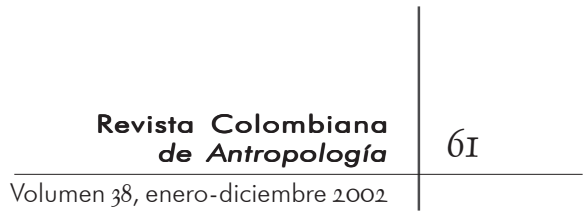

plementación de un plan de organización y manejo para el área núcleo ${ }^{16}$ de la reserva -que había sido definida dentro de otra figura conservacionista, el parque nacional natural Sierra Nevada de Santa Marta- ${ }^{17}$. La FPSNSM inició su tarea fundamentada en nociones como las siguientes, que no varían mucho de las nociones clásicas de la etnografía serrana:

La SNSM alberga tres grupos indígenas: Kogi, Ika, y Sanha, que habitan en Resguardos. El manejo respetuoso del medio ambiente por parte de estos grupos indígenas responde a una tradición milenaria de una de las culturas más desarrolladas de América -la cultura Taironabasada en la explotación de una gran variedad de recursos de las diferentes zonas altitudinales (Unesco-FPSNSM, 1987: 3-4).

En este plan, la Sierra se reconoció como un lugar de refugio de poblaciones marginales, y sus problemáticas socioeconómicas se vincularon a la degradación ambiental (FPSNSM, I997: xiv-xv). Los objetivos centrales de la estrategia de conservación se definieron de I7. El parque como tal fue establecido en 1964 y ampliado en 1977. Cubre 383 mil hectáreas, dentro de las cuales está, además, el resguardo indígena Kogui-Malayo. acuerdo con la etnicidad de los grupos de población de base de la Sierra: "problemas campesinos" y problemas "indígenas". Como solución predominó en el discurso la imagen ambiental de los kogui: "Fue posible reconocer la importancia de las culturas indígenas tradicionales, y cómo ellas constituyen la mejor opción y garantía para el manejo ambiental y territorial de la Sierra Nevada a partir de sus conocimientos ancestrales" (FPSNSM, I997: xvi). Basada en estas ideas, a principios de los años I990 la Fundación elaboró un Plan diagnóstico integral regional (FPSNSM, I997), cuyos resultados se incorporaron a la figura del Plan Nacional de Rehabilitación, y así Pro Sierra fue encargada de desarrollar lo que se ha llegado a conocer desde ese entonces como la estrategia de conservación para la Sierra Nevada de Santa Marta, de la cual hace parte el plan de desarrollo sostenible de la SNSM.

De esta manera, las nociones sobre los kogui, heredadas de Preuss, Reichel-Dolmatoff y demás antropólogos, se institucionalizaron como solución a una problemática ambiental y se transmitieron en discursos nacionales e internacionales (véase Mayr, 1995). En I998 el trabajo de la FPSNSM recibió legitimación estatal y nacional mediante el nombramiento de Juan Mayr como minis- 
tro del Medio Ambiente del gobierno del presidente Andrés Pastrana (1998-2002). Ahora, las interpretaciones antropológicas se transforman en políticas institucionales o estatales y entran a formar parte de la estructura gubernamental de clasificación y reorganización espacial ${ }^{18}$. Sus implicaciones se traducen en conflictos de autoridad sobre el espacio o sobre la producción y negociación de la identidad y otros procesos de negociación y circulación de poder (Brosius, 1997, I999; Escobar, I999; Moore, 1997). Finalmente, aquellas representaciones, que como hemos visto son resultado de momentos y procesos históricos, se legitiman por medio del uso de herramientas y técnicas que las visualizan, que las hacen legibles. Las mismas han llevado a algunos autores a cuestionar y reconocer este proceso que establece una relación entre lo técnico y lo político (Brosius y Russell, 200I).

\section{ORGANIZACIÓN TECNOCIENTÍFICA}

$$
\text { ( }
$$

OMO RESULTADO DEL ENMARAÑAMIENTO CONTEMPORÁNEO ENTRE LO político, lo tecnológico y lo biológico, las ciencias de la comunicación y la biología moderna han construido un lenguaje común para la clasificación de las nuevas formas de datos científicos. El nacimiento de la biodiver-

I8. Uno de los grandes dilemas de la conservación de la biodiversidad es la yuxtaposición de áreas silvestres y frágiles con sus habitantes. La reubicación, la compra de tierras, etcétera, son soluciones que no sólo han sido infructuosas sino que, además, han creado grandes conflictos sociales. En el caso colombiano, el sistema legal ha querido mitigar esto con el reconocimiento al libre desarrollo de las comunidades tradicionales que habitan estos territorios (decreto 622 de 1977 , artículo $7^{\circ}$, en Arango y Sánchez, 1998: 234). Sin embargo, la experiencia ha mostrado que existen conflictos de intereses (véase, por ejemplo, Ulloa et al., I996) en estos espacios; los conflictos, por supuesto, varían, y en el caso de la SNSM forman parte de la historia de la articulación de discursos y representaciones en proceso. sidad y, más aún, su principio global y deterritorializado necesitó de aparatos y técnicas de medición, visualización y clasificación generalizables, que resultan en nuevas formas de organización (Brosius y Russell, 200I; Escobar, I999; Haraway, 1997). En el caso de la Sierra Nevada, el lenguaje clasificatorio principal ha sido la evaluación ecológica rápida (EER), una herramienta muy común en el establecimiento de diagnósticos para estrategias de conservación.

Esta herramienta se apoya en la formulación de mapas digitales -sistemas de información geográfica (SIG)- que resultan del cruce de información socioeconómica y políticoadministrativa con 


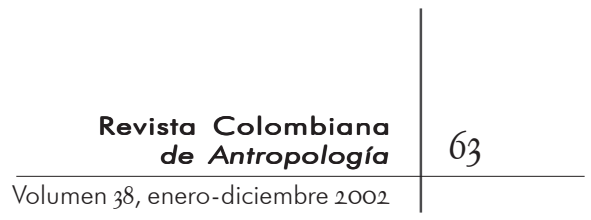

información biogeográfica; la información se incorpora luego a una base de datos de SIG, en la que se determinan niveles y áreas críticas. Finalmente, los resultados se correlacionan con las entidades y figuras sociopolítico-administrativas para crear un modelo de manejo coordinado entre las diferentes figuras administrativas y la información de la evaluación ecológica rápida.

Por medio de la EER se produjo una serie de mapas que dividen a la Sierra a partir de las llamadas áreas críticas, que tienen valores de riesgo asignados (FPSNSM, 1998). De esta manera, el territorio y el espacio se reorganizan en categorías de riesgo; de esta clasificación pueden depender asuntos como inversión, investigación, planes de reubicación, apoyo de ciertas prácticas culturales y control de otras.

Mediante la observación satelital, la reproducción digital del espacio ha materializado una historia de procesos e interpretaciones sociales en imágenes surreales que condensan la problemática social y ambiental de la Sierra Nevada. Estas imágenes redefinen el espacio en lo que Haraway (I997) ha llamado entidades semiótico-materiales -formas concretas que localizan y dan sustancia, sistemas posmodernos que surgen de la interacción entre lo biológico y lo tecnológico, en formas contemporáneas en las que lo cultural y lo natural se constituyen mutuamente-.

Así, las ideas sobre la situación de marginalidad de la Sierra se consolidaron en términos globales, en un lenguaje tecnocientífico que las hace parte de un discurso -la biología de la conservación$\mathrm{y}$, al mismo tiempo, las reconoce e incluye físicamente en este espacio. No es entonces una metáfora sino una forma de representación híbrida en la que representaciones, imágenes y formas tecnocientíficas, localidades, lugares e, incluso, niveles de riesgo, así como los kogui, junto con los demás habitantes del macizo, fueron incorporados "[é]tica, así como existencial y materialmente en tecnologías y prótesis tecnológicas” (Fischer, I999: 467).

Por medio de las herramientas del discurso conservacionista los habitantes de la Sierra Nevada entraron en contacto directo con un Otro, representado por el conocimiento especializado que los define y legitima a la vez. La imagen de lo kogui, que inicialmente legitimaba y en cierto sentido movilizaba personas para su propia conservación, ha sido reincorporada por el aparato de la conservación. Así, se ve envuelta en procesos alternativos de re- 
interpretación y reproducción que sugieren una sensibilidad a las sutilezas y complejidades que surgen en la marginalidad.

\section{Conservación ALTERNATtVA}

N

I CONTACTO MÁS DIRECTO CON LA IMAGEN KOGUI HA SIDO EN LA cuenca del Buritaca. Relacionada con los procesos sociales 1 y políticos resultado de los proyectos arqueológicos y biológicos en torno a Ciudad Perdida, la cuenca tiene una historia de recientes migraciones y desplazamientos de indígenas, campesinos, investigadores y grupos armados. Como tal, es un lugar donde es posible observar cómo las personas desde diversas posiciones "[a]ctivamente enfrentan su marginalidad al protestar, reinterpretar y adornar su exclusión” (Tsing, 1993: 5).

Alto de Mira es un sitio arqueológico ubicado entre las cuencas del Buritaca y el Guacacha, en el que pasé un par de noches en camino entre Ciudad Perdida y Santa Marta. Después de un ascenso largo, la vista sobre el resto del valle es más que gratificante; hay un par de casas para dos o tres trabajadores $\mathrm{y}$ otras para una o dos familias kogui que a veces pasan temporadas allá. Aquí se construyó la primera estación de campo de la FPSNSM, concebida inicialmente como base de estudios biológicos y ecológicos.

En los primeros años de la Fundación allí se realizaron algunos proyectos de investigación bajo el supuesto de la participación y cooperación con la comunidad. Sin embargo, la misma no se obtuvo en todos los casos, pues hubo resistencia por parte de familias kogui de la zona. Esta aparente contradicción me la aclaró un mama cuando, para explicarme por qué no eran viables los proyectos biológicos, me contó la historia de un proyecto botánico realizado en Alto de Mira que, literalmente, nunca floreció, pues los indígenas no dejaron: arrancaban las marquillas de conteo e identificación de las palmas cuando pasaban por las parcelas de levantamiento botánico. Los indígenas enfrentaron otros proyectos de forma más directa, como en el caso del proyecto de control de pacas (Agouti sp.), que implicaba colocarle collares de rastreo a los animales; otros se descartaron desde su formulación bajo la idea de que la preservación de los animales depende en gran parte del control espiritual de los mamas, del uso correcto de los pagamentos, y no de las prácticas materiales occidentales. Este tipo de acciones y argumentos fueron los que, 


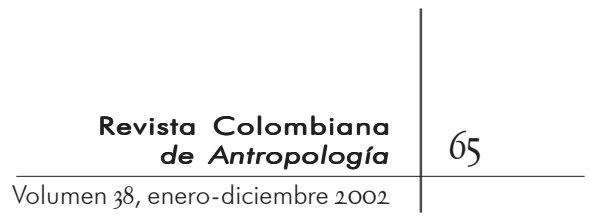

finalmente, llevaron a personas como Ricardo Rey, director de la estación por muchos años, a revaluar la idea y los procesos de investigación biológica orientados a la conservación (entrevista con Ricardo Rey, 200I).

Sin embargo, para él esto no ha sido en vano, pues considera que los actos de oposición por parte de los indígenas de la zona lo llevaron a repensar y de cierta manera obligaron a los miembros de la Fundación a reorientar su mirada de los bosques a sus habitantes. De esta manera se inició un proceso de aprendizaje sobre los significados indígenas de Alto de Mira. Los mamas empezaron a contar la historia del sitio, a hablar de sus significados y a enseñar, en palabras de Rey, que este es un lugar lleno de vida espiritual y que las acciones e imposiciones occidentales lo estaban contaminando. Se inició entonces un proceso de transformación por parte de algunos miembros de la institución que incluyó la suspensión de investigaciones (científicas) allí.

Como resultado de este proceso, herramientas y prácticas -occidentales- para la conservación de la biodiversidad empezaron a considerarse insuficientes; en cambio, se estimó que aprender del Otro, del indígena kogui, era lo que finalmente se requería para cumplir el gran objetivo de salvar o preservar a la Sierra, sus ecosistemas y culturas. La resistencia a estos proyectos de investigación científica por medio de prácticas y acciones sutiles generó un proceso que estableció nuevas formas de entender los bosques -y con esto, podría decirse que una forma alternativa de aproximarse a la conservación-.

En la oficina de la Fundación Pro Sierra en Santa Marta, Ricardo expresó así esta transformación. Decidí editar y transcribir varios apartes de nuestra conversación, pues juntos agrupan las diferentes fases de este proceso y sus resultados:

-[Hablando sobre las características de Alto de Mira] No todos los sitios tienen como esa energía, esa parte que todavía ellos reciben que es vital para ellos. De pronto, por ejemplo, espiritualmente algo que esté pasando aquí [en Santa Marta], en el parque Tairona, en Pueblito, o en otro sitio [tiene que ver mucho con] Ciudad Perdida o Alto de Mira. De pronto para arreglar una enfermedad aquí abajo hay que ir a hacer el pago allá. Eso implica unos cambios; hay que irse a lo indígena, que no se contamine, que se respete, implica que se mantenga por sí solo, pero se hace el trabajo allá pero se está 
haciendo para otro sitio. Desde allá para otro sitio. Entonces el espacio se vuelve una cosa que no tiene fronteras (...)

Entonces [los mamas], dijeron en un momento dado que paráramos un poco de estar ahí, que eso tiene su tiempo que se cumple, y realmente tiene que cerrarse. Por otro lado pidieron hacerle un trabajo de purificación al sitio, hacerle una mortuoria al sitio, hacerle unos pagos espirituales; tomar algunos puntos de referencia y hacer el pago, es decir, la ley indígena, que es lo que argumenta el sitio. Esto purifica el sitio; por ejemplo, en Alto de Mira eso ya se hizo. Dejarlo un poco quieto, dejarlo un poco solo, escuchar qué está saliendo de los adivinos, conocer el adivino qué dice del árbol, qué dice Kalxsé [Padre del bosque], quién no paga, qué no se puede cortar, qué no se puede mirar, qué no se puede cazar, en fin, eso implica estar un poco quieto escuchándolos. Pero eso actualmente le ha servido al sitio. El sitio tiene más fuerza, internamente, espiritualmente tiene más fuerza.

No obstante,

[Alto de Mira] sigue siendo la estación ecológica para la Fundación. Pero con un manejo diferente, con un control diferente, más espiritual. Pero eso ha alimentado mucho el sitio; el sitio espiritualmente tiene mucha fuerza. Hay más animales, más aves, hay más árboles, más bosque, más culebra, se pararon un poco los derrumbes, huracanes y otros problemas ambientales. Entonces, viéndolo hoy en día yo lo veo muy positivo, haber escuchado eso, haberle prestado la atención; y si uno quiere hacer cualquier proyecto tiene que hacer la consulta con ellos.

Con esta nueva consideración que se derivó de haber escuchado a los indígenas, la idea y forma de concebir los proyectos ha ido adquiriendo un nuevo significado: ahora la palabra proyecto se asemeja más a escuchar a los indígenas, a escuchar a los lugares. Por lo menos así lo es para algunos, y sólo algunos, miembros de Pro Sierra; el resultado ha sido cierta tensión en la red burocrática de formulación y ejecución de proyectos dentro de la institución. En este sentido, se yuxtaponen dos formas de entender los proyectos: una, ligada al proceso científico y acorde con los preceptos del aparato de la conservación -por lo general se encuentra en los archivadores de la formulación de proyectos y en las publicaciones de informes de trabajos realizados en zonas de mayoría campesina-. La otra se asemeja más a una forma alternativa de conservación, y está basada en preceptos indígenas; se ha aplicado sólo en un lugar, Alto de Mira, y en 


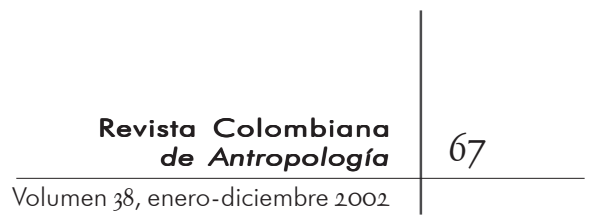

la estructura de la Fundación se ve más como una iniciativa de Ricardo Rey que como un proyecto de la Fundación. Sin embargo, para ese sitio siguen vetadas las posibilidades de realizar cualquier tipo de proyecto -en el sentido occidental de la palabra-.

Esta nueva manera de concebir la investigación y las acciones sobre conservación se convierten en elementos sutiles pero importantes al considerarlos dentro de los procesos burocráticos en los que se ha visto inmersa la Fundación. Según varias personas que trabajan -o han trabajado- allí hace mucho tiempo, la entidad ha crecido a tal punto que las presiones y políticas del aparato de la conservación la han, prácticamente, sobrecogido. Como resultado, los motivos originales que llevaron a la creación de la FPSNSM se han diluido en el discurso global de la conservación (conversaciones con Sylvia Botero, Juana Londoño y Ricardo Rey, junio de 200I); las presiones de los donantes internacionales, el estado y las instituciones y organizaciones locales han creado un contexto en el que los diferentes intereses se confrontan y se integran al proceso de burocratización y poder en esas instituciones. Como se decía antes, esto lleva a que se creen espacios y formas burocráticas para mantener esa imagen, ese sujeto, esa representación. Pero ese mismo sujeto puede convertirse en un elemento que introduce tensión dentro del proceso burocrático y el desarrollo de la institución.

En este caso, el elemento surge de un proceso en el cual las imágenes del Otro se expresan en combinación con interpretaciones aprendidas, en un escenario lleno de imágenes y significados que resultaron de procesos históricos de la historia del Buritaca. El significado que el sitio Alto de Mira ha adquirido para ciertos miembros de la Fundación puede leerse entonces como un proceso de copia e imitación de las representaciones (Taussig, 1993). En este caso, el juego de las representaciones se confunde en una doble-vía, a tal punto que el sitio como tal es entendido a partir de nociones indígenas que, a su vez, contienen elementos de una historia larga de representaciones y negociaciones. Así, es un proceso en el que se han mezclado formas de conocimiento occidentales e indígenas y hoy se exponen como formas híbridas para la negociación de(desde) la marginalidad.

Este proceso de producción de una imagen del Otro se ha articulado de manera compleja con un discurso de orden global, y como consecuencia ha hecho visible el uso de la reinterpretación de representaciones como formas alternativas de conservación 
dentro de una estructura convencional de instituciones ambientalistas. Irónicamente, dentro de la misma institución cuya estructura burocrática mayor la ha llevado a un proceso en el que las organizaciones indígenas han cuestionando proyectos de conservación de gran escala, que la Fundación representa en nombre de grandes donantes internacionales.

En junio de 200I, cuando llegué a la casa indígena de Santa Marta, encontré un ambiente tenso. Miembros de la unidad de parques del Ministerio del Medio Ambiente y de la Fundación Pro Sierra entraban y salían de reuniones con los líderes indígenas. Era un momento intenso de un proceso que se había iniciado a finales de 2000, cuando la Organización territorial de cabildos de la Sierra Nevada de Santa Marta se opuso a la realización por parte de la Fundación Pro Sierra de unos proyectos de conservación, desarrollo sostenible y manejo participativo de recursos naturales. Los indígenas se oponían a partir de reclamos sobre la desatención y el desconocimiento de su autoridad y autonomía en estos procesos. Estas críticas que formulaba la organización indígena con respeto a sus formas de autoridad están sustentadas por diferentes reconocimientos a la diversidad -como el reconocimiento constitucional de la autoridad local de las comunidades indígenas en sus territorios, el derecho de representatividad política con curules asignadas en el Congreso y el conocimiento ambiental indígena, entre otros-; esto dio más legitimidad y complejidad al asunto. Con los proyectos suspendidos temporalmente se inició un proceso entre la Fundación, las organizaciones indígenas y otras entidades gubernamentales, privadas, nacionales y extranjeras, para buscar soluciones compartidas.

De manera silenciosa, una de las grandes participantes en la controversia ha sido esta historia de representaciones y sus múltiples legitimaciones: el énfasis sobre el conocimiento indígena concebido en términos ambientales -en el que se conjugan la sacralidad y la tradición que lo fortalecen- legitimó la necesidad de articulación con el discurso de la conservación. Así nació Pro Sierra. En este proceso las representaciones se han institucionalizado como política gubernamental y por medio de herramientas y formas de visualización tecnocientíficas fueron clasificadas e incluidas en el espacio transnacional de la conservación de la biodiversidad. Esto, a su vez, ha legitimado la intervención del aparato de la conservación en estos contextos, 


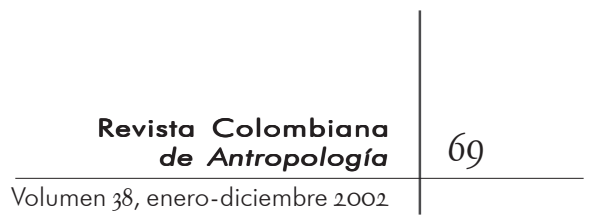

generando conflictos sobre la autonomía y la autoridad local.

Cuestionar la unidad u homogeneidad de la cultura permite aproximarse a una serie de sutilezas críticas. Problematizar la producción etnográfica de gente y lugar, y de hacerlo aplicar una dosis de reflexividad a nuestra propia práctica permite seguir el discurso etnográfico en sus múltiples desarrollos. En este caso, como lo sugiere Appaduari (I996: I82), abre una nueva forma para pensar la compleja coproducción entre categorías indígenas e intelectuales, administradores y etnólogos, a las que agregaría científicos y las nuevas ficciones y formas de clasificación y re-producción tecnocientíficas. Identificar el conocimiento antropológico y su papel como un elemento más en una serie de canales de representaciones permite hacer seguimientos que muestran la complejidad de articulaciones con otros discursos en los que las representaciones actúan en múltiples formas. Es en este sentido que he querido leer este proceso de producción cultural de lo kogui.

\section{BibliografíA}

Álvarez, RoBert A. 1995. "The Mexican-US border: The making of an Anthropology of Borderlands". Annual Review of Anthropology. 24.

Appadurai, Arjun. 1998. Modernity at Large. University of Minnesota Press.

Arango, Raúl y E. Sánchez. 1997. Los pueblos indígenas de Colombia 1997. Desarrollo y territorio. Departamento Nacional de Planeación/TM Editores. Bogotá.

Århem, Kaj. 1993. "Ecosofía Makuna”. En François Correa (editor). La selva humanizada. Ecología alternativa en el trópico húmedo colombiano. Cerec-Instituto Colombiano de Antropología. Bogotá.

Bocarejo, Diana. 200I. "Tradición milenaria: dinamismo y vigencia de la tradición indígena en la Sierra Nevada de Santa Marta". Tesis de antropología, sin publicar. Universidad de Los Andes. Bogotá.

Brosius, J. Peter. 1997. "Endangered Forests, Endangered People: Environmentalist Representations of Indigenous Knowledge". Human Ecology. 25: I. 
-1999. "Analysis and Interventions. Anthropological Engagements with Environmentalism". Current Anthropology. 40: 3.

200I. "Local Knowledges, Global Claims: On the significance of Indigenous ecologies in Sarawak, East Malaysia". En Indigenous Traditions and Ecology. Harvard University Press/the Center for the Study of World Religions. Cambridge, Mass.

Brosius, J. Peter y D. Russell. 200I. "Conservation from Above: An anthropological perspective on transboundary protected areas and ecoregional planning". Manuscrito sin publicar.

CÁrdenas TÁmara, Felipe. 2000. Antropología y ambiente: enfoques para una comprensión de la relación ecosistema-cultura. Ambiente y Desarrollo, serie Investigación 5. Pontificia Universidad Javeriana. Bogotá.

CORONEL, VAleria. 2000. "Conversión de una región periférica en localidad global: actores e implicaciones del proyecto culturalista en la Sierra Nevada de Santa Marta”. En Antropologías transeúntes. Eduardo Restrepo y María Victoria Uribe (editores). ICANH. Bogotá.

Descola, Philippe. 1996. "Constructing Natures. Symbolic ecology and social practice". En Nature and Society. Anthropological Perspectives. Philippe Descola \& Gilsi Pálsson (editores). Routledge. Londres y Nueva York.

Dussán de Reichel-Dolmatoff, Alicia. 1965. "Problemas y necesidades de la investigación etnológica en Colombia”. Antropología 3. Ediciones Universidad de Los Andes. Bogotá.

Escobar, Arturo. 1996. "Constructing nature: Elements for a poststructural political ecology”. En R. Peet y M. Watts (editores). Liberation ecologies: Environment, development, social movements. Routledge. Londres.

1999. El final del salvaje. Naturaleza, cultura y política en la antropología contemporánea. Cerec-Ican. Bogotá.

FigueroA, José A. 1998. "Excluidos y exiliados: indígenas e intelectuales modernistas en la Sierra Nevada de Santa Marta”. En Modernidad, identidad y desarrollo. María Sotomayor (editora). Ican. Bogotá.

Fischer, Michael J. I999. "Emergent Forms of Life: Anthropologies of Late or Postmodernities". Annual Review of Anthropology. 28.

Fortun, Kim. 1999. "Locating Corporate Environmentalism. Synthetics, Implosions, and the Bophal disaster". En Critical Anthropology Now. Unexpected contexts, shifting constituencies, changing agendas. George Marcus (editor). School of American Research. Santa Fe. 


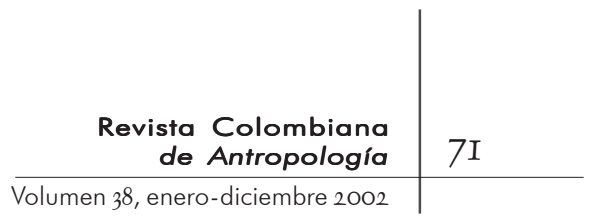

Fundación Pro-Sierra Nevada de Santa Marta. i997. Plan de desarrollo sostenible de la Sierra Nevada de Santa Marta. Estrategia de conservación de la SNSM. Proyecto de cooperación colombo-alemán. Fundación Pro-Sierra Nevada de Santa MartaMinisterio del Medio Ambiente-Departamento Nacional de Planeación.

I998. “Evaluación ecológica rápida. Definición de áreas críticas para la conservación en la Sierra Nevada de Santa Marta. Colombia”. Fundación Pro-Sierra Nevada de Santa MartaMinisterio del Medio Ambiente-The Nature Conservancy-USAID.

s. f. "Problems that led to the Collaborative Management Agreements”. Documento inédito. Fundación Pro-Sierra Nevada de Santa Marta. Santa Marta.

Giraldo, Santiago. I999. "A History of Tairona Archaeology in Colombia, I920-I980: From Culture History to archaeology as Politics”. Tesis de maestría. The University of Chicago. Chicago.

Gupta, Akhil y J. Ferguson. i997. "Culture, Power, Place: Ethnography and the End of an Era”. En Culture, Power, Place. Explorations in Critical Anthropology. A. Gupta y J. Ferguson (editores). Duke University Press. Durham.

Haraway, Donna. I991. Symians, Cyborgs, and Women. The reinvention of nature. Routledge. Nueva York.

I997.Modest_Witness@Second_Millenium.FemaleMan Meets_OncoMouse ${ }^{\overline{\mathrm{TM}}}$ : Feminism and Technoscience. Routledge. Nueva York.

Herrera de Turbay, Luisa F. I985. Agricultura aborigen y cambios de vegetación en la Sierra Nevada de Santa Marta. Fundación de Investigaciones Arqueológicas Nacionales-Banco de la República. Bogotá.

Hugh-Jones, Christine. 1979. From the Milk River: Spatial and Temporal Processes in Northwest Amazonia. Cambridge University Press. Cambridge.

Hugh-Jones, Stephen. I979. The Palm and the Pleiades: Initiation and Cosmology in Northwest Amazonia. Cambridge University Press. Cambridge,

Hurtado, Andrés. I984. "Réquiem por la Sierra Nevada”. El Tiempo. Bogotá. I2 de febrero: 4-B.

Jackson, Jean. I983. The Fish People: Linguistic Exogamy and Tukanoan Identity in Northwest Amazonia. Cambridge University Press. Nueva York.

Koepring, Klaus-Peter. I983. Adolf Bastian and the psychic unity of 
mankind: the foundations of anthropology in nineteenth century Germany. University of Queensaland Press. Nueva York.

Latour, Bruno. I993. We have never been modern. Harvard University Press. Cambridge.

Legast, Anne. 1986. El animal en el mundo mítico Tairona. Banco de la República. Bogotá.

Marcus, George. 1999. "Critical Anthropology Now. An introduction": Unexpected contexts, shifting constituencies, changing agendas. School of American Research Press. Santa Fe.

Marcus, George y M. Fischer. 1999 [1986]. Anthropology as Cultural Critique. An experimental moment in the human sciences. Segunda edición The University of Chicago Press. Chicago.

Mason, Gregory. 1940. South of Yesterday. Henry Holt and Co. Nueva York.

MaYr, JuAn. I995. "Case study: Colombia”. Ponencia presentada en la American Association for the Advancement of Science (AAAS) International Directorate's Program on Population and Sustainable Development (PSD): www.aaas.org/international/ psd/biod.maldon.htm

Moore, Donald. 1997. "Remapping resistance. 'Ground for struggle' and the politics of place". En Geographies of resistance. S. Pile y M. Keith (editores). Routledge. Londres.

Nicholas, Francis C. I9OI. "The aborigines of the Province of Santa Marta, Colombia”. American Anthropologist. 3: 4.

Orrantia, Juan C. 2002. "Esencialismo desde el Corazón del Mundo. Información como legitimación del riesgo". Revista de Antropología y Arqueología. I3. Universidad de Los Andes. Bogotá.

Pineda Camacho, Roberto. 20or. Sociedades heridas: el desafío de una nación multicultural en un escenario de conflicto. Documento 7. Centro de Estudios Socioculturales e Internacionales (Ceso). Facultad de Ciencias Sociales de la Universidad de Los Andes. Bogotá.

Pratt, Mary Louise. 1992. Imperial Eyes. Travel Writing and Transculturation. Routledge. Nueva York.

Preuss, Konrad Th. I993 [1916]. Visita a los indígenas Kággaba de la Sierra Nevada de Santa Marta. Observaciones, recopilación de textos y estudios lingüísticos. Parte I y II. Colcutura-Ican. Bogotá.

Ramos, Alcida Rita. 1998. Indigenism. Ethnic Politics in Brazil. The University of Wisconsin Press. Madison.

Reichel-Dolmatoff, Gerardo. I975. “Templos Kogi. Introducción al 


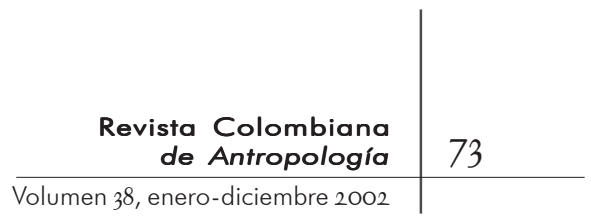

simbolismo y a la astronomía del espacio sagrado. Revista Colombiana de Antropología. I9. 1976a. "Cosmology as ecological analysis: a view from the rainforest". Man 2: 3 . I976b. "Training for priesthood among the Kogi of Colombia". En Johanes Wilbert (editor). Enculturation in Latin America: An Anthology. University of California Press. Los Angeles. 1978. "The Loom of Life: A Kogi principal of integration". Journal of Latin American Lore. 4: I. 1982a. "Cultural change and environmental awareness: a case study of the Sierra Nevada de Santa Marta, Colombia". Mountain Research and Development. 2: 3.

1982b. "Astronomical models of social behavior among some Indians of Colombia". En Anthony Aveni and Gary Urton (editores). Ethnoastronomy and Archeoastronomy in the American Tropics. Annals of the New York Academy of Science. Volumen 385. 1985. Los Kogi. Una tribu de la Sierra Nevada de Santa Marta. Segunda edición. Tomos I y II. Nueva Biblioteca Colombiana de Cultura. Procultura. Bogotá. I99I. Indios de Colombia. Villegas Editores. Bogotá.

Serje, Margarita. I986. "Agricultura y urbanismo en la cultura tairona”. Boletín del Museo del Oro. I9. Banco de la República. Bogotá.

Soto, Álvaro. I975. "Introducción al programa de estaciones antropológicas". Revista Colombiana de Antropología. i8.

I988a. "Primer balance sobre Ciudad Perdida. Tairona, al rescate de una civilización”. El Tiempo, Lecturas Dominicales. Bogotá. I7 de abril. 1988b. La Ciudad Perdida de los tairona. Historia de su hallazgo y descubrimiento. Neotrópico. Bogotá.

Taussig, Michael. 1993. Mimesis and Alterity. A Particular History of the Senses. Routledge. Nueva York.

Tsing, Anna L. I993. In the Realm of the Diamond Queen. Marginality in an Out-of-the- Way Place. Princeton University Press. Princeton.

UlloA, Astrid. 200I. "El nativo ecológico. Movimientos indígenas y medio ambiente en Colombia". En Movimientos sociales, estado y democracia en Colombia. Mauricio Archila y Mauricio Pardo (editores). Ican-CES-Universidad Nacional de Colombia. Bogotá. 
Ulloa, Astrid, H. Rubio y C. Campos. 1996. Trua Wuandra. Estrategias para el manejo de fauna con comunidades embera en el parque nacional Utría, Chocó, Colombia. Orewa-Fundación NaturaMinisterio del Medio Ambiente-OEI. Bogotá.

Unesco. 200I. Unesco. Man and the Biosphere Program. www.unesco.org/mab/brfaq.htm

Unesco-Fundación Pro-Sierra Nevada de Santa Marta. 1987. "Plan de ordenamiento y manejo integral de las cuencas altas de los ríos Buritaca y Guachaca-Parque Natural Sierra Nevada de Santa Marta”. Documento inédito. Fundación Pro-Sierra Nevada de Santa Marta.

Uribe, Carlos. A. I988. "De la Sierra Nevada de Santa Marta, sus ecosistemas, indígenas y antropólogos". Revista de Antropología. Universidad de los Andes. IV: I. Bogotá.

1990. "We the Elder Brothers: Continuity and Change amongst the Kággabba of the Sierra Nevada de Santa Marta, Colombia”. Disertación doctoral. Universidad de Pittsburgh.

I993. "Konrad Theodor Preuss y la Sierra Nevada de Santa Marta". En Visita a los indígenas Kággaba de la Sierra Nevada de Santa Marta. Observaciones, recopilación de textos y estudios lingüísticos. K. Preuss. Parte I. Colcltura-Ican. Bogotá.

I998. "De la vitalidad de nuestros hermanos mayores de la nevada”. Revista de Antropología y Arqueología. Iо: 2. Universidad de Los Andes. Bogotá.

Viveiros de Castro, Eduardo. 1996. "Images of nature and society in Amazonian ethnology". Annual Review of Anthropology. 25.

Vollmer, Loraine. 1983. "La Ciudad Botánica". Manuscrito sin publicar.

\section{Prensa}

\section{El Tiempo}

197I. "La cultura Tairona. Donde el tiempo no existe". 6 de julio. 1976a. "Murallas para proteger tesoros piden indígenas". Io de junio.

I976b. "Foro indígena para proteger ciudad sagrada". I4 de junio. 


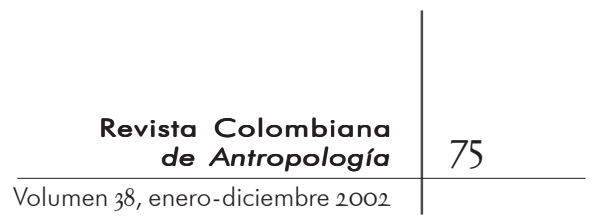

\section{El Espectador}

I968. "Indígenas de Sierra Nevada se extinguen”. I6 de diciembre. I982. "iQue se abran las puertas!". 25-28 de julio. I983. "Ciudad Perdida. Mucho ruido y pocas nueces". 30 de agosto.

\section{Entrevistas personales (junio-julio de 200I)}

Sylvia Botero.

Juana Londoño.

Ricardo Rey.

Álvaro Soto. 